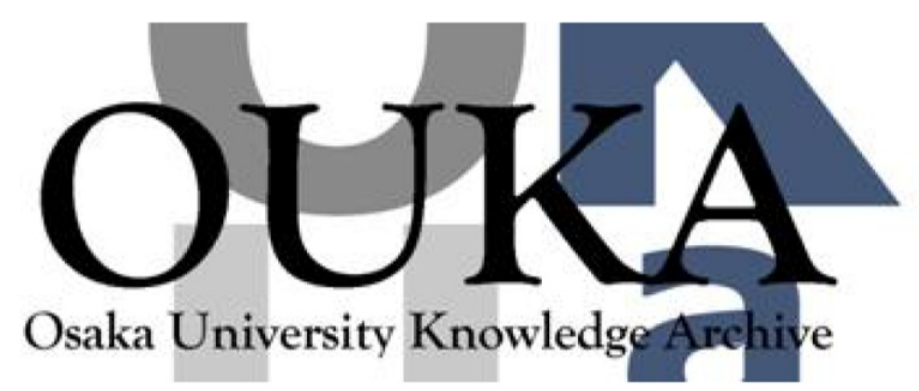

\begin{tabular}{|c|l|}
\hline Title & $\begin{array}{l}\text { Guest-host electro-optic switching in spin- } \\
\text { coated polymer ferroelectric liquid crystal } \\
\text { film }\end{array}$ \\
\hline Author(s) & $\begin{array}{l}\text { Okazaki, Shoji; Uto, Sadahito; Ozaki, Masanori } \\
\text { et al. }\end{array}$ \\
\hline Citation & Applied Physics Letters. 71(23) p. 3373-p. 3375 \\
\hline Issue Date & $1997-12-08$ \\
\hline oaire:version & VoR \\
\hline URL & https://hdl. handle.net/11094/75843 \\
\hline rights & \\
\hline Note & \\
\hline
\end{tabular}

Osaka University Knowledge Archive : OUKA

https://ir. Library. osaka-u. ac. jp/

Osaka University 


\section{Guest-host electro-optic switching in spin- coated polymer ferroelectric liquid crystal film}

Cite as: Appl. Phys. Lett. 71, 3373 (1997); https://doi.org/10.1063/1.120399

Submitted: 05 May 1997 . Accepted: 26 September 1997 . Published Online: 04 June 1998

Shoji Okazaki, Sadahito Uto, Masanori Ozaki, Katsumi Yoshino, Kent Skarp, and Bertil Helgee

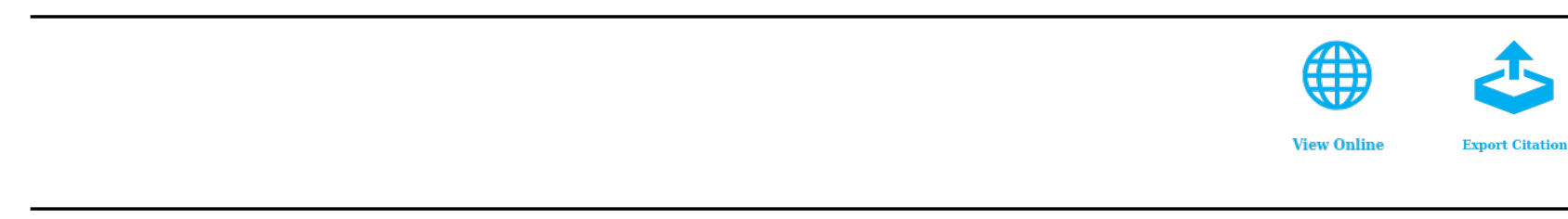

\section{ARTICLES YOU MAY BE INTERESTED IN}

Energy efficient plasma processing of gaseous emission using a short pulse discharge Applied Physics Letters 71, 3364 (1997); https://doi.org/10.1063/1.120338

Thermal stability of amorphous hard carbon films produced by cathodic arc deposition Applied Physics Letters 71, 3367 (1997); https://doi.org/10.1063/1.120339

Spontaneous formation and photoluminescence of ZnSe dot arrays

Applied Physics Letters 71, 3370 (1997); https://doi.org/10.1063/1.120340

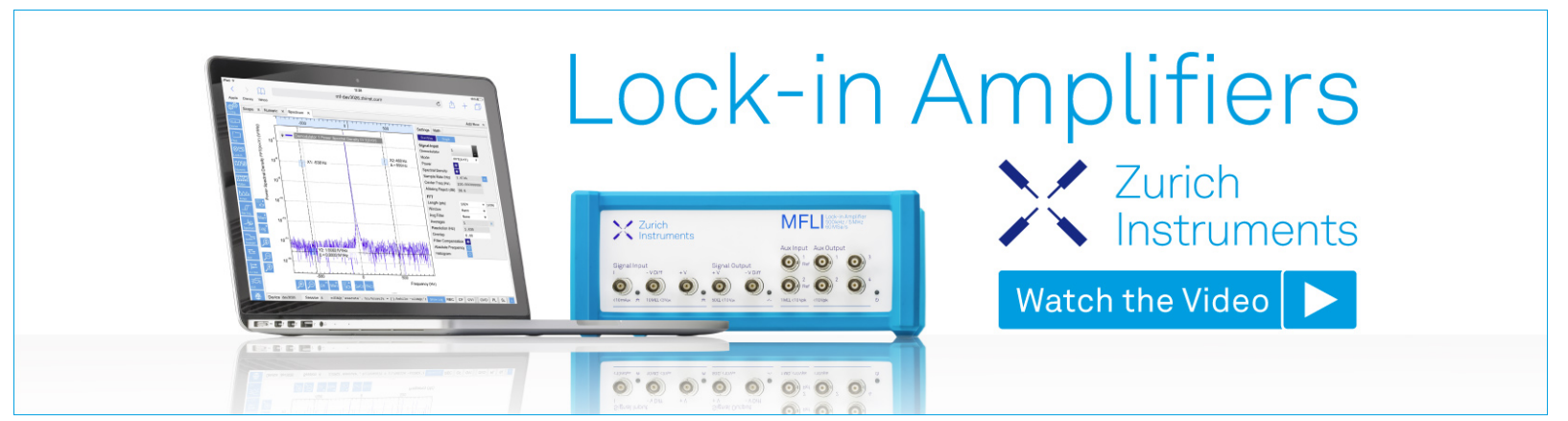




\title{
Guest-host electro-optic switching in spin-coated polymer ferroelectric liquid crystal film
}

\author{
Shoji Okazaki, Sadahito Uto, Masanori Ozaki, and Katsumi Yoshino a) \\ Department of Electronic Engineering, Faculty of Engineering, Osaka University 2-1 Yamada-Oka, Suita, \\ Osaka 565, Japan \\ Kent Skarp \\ Department of Physics, Chalmers University of Technology, S-412 96 Göteborg, Sweden
}

Bertil Helgee

Department of Polymer Technology, Chalmers University of Technology, S-412 96 Göteborg, Sweden

(Received 5 May 1997; accepted for publication 26 September 1997)

We have proposed an electro-optic effect in dichroic-dye-doped polymer ferroelectric liquid crystal thin films prepared by a spin-coating technique. A high-quality homeotropically aligned film has been realized. Guest-host type electro-optic switching is confirmed in the dye-doped film, and basic properties such as electric field dependence of the response time are studied. (C) 1997 American Institute of Physics. [S0003-6951(97)03947-8]

Polymer liquid crystals have attracted considerable attention from fundamental and practical points of view because of their excellent mechanical properties, processability, and the possibility of functional application. The application of polymer liquid crystals, however, has been restricted to a nonoptical use and optoelectronic devices using this material have never been realized, since polymer liquid crystals have a markedly high viscosity and the driving force by an applied electric field originating from dielectric anisotropy is small. Polymer ferroelectric liquid crystals (PFLC) show a fast electro-optic switching because of the strong interaction between the spontaneous polarization and the electric field, so that electro-optic devices using PFLC are expected to be realized in the near future. ${ }^{1,2}$

For the application of polymer liquid crystals to electrooptic devices, a conventional cell geometry in the same way as used in low molecular weight liquid crystal display devices, in which the liquid crystal is sandwiched between two glass plates or two flexible polymer films, has been used. We proposed a novel preparation technique of liquid-crystallinepolymer thin films using spin coating from a solution of PFLC and have reported their electro-optic properties. ${ }^{3-6}$ Using this film preparation technique, a homeotropically aligned PFLC thin film of good quality with large area can be easily prepared. Moreover, the spin-coated PFLC film has asymmetric surface conditions and one can expect interesting surface effects on the electro-optic response and the phase transition properties of the film. In this letter, a new type of electro-optic effect in a dye-doped PFLC is proposed.

A sidechaintype ferroelectric liquid crystal with a backbone of a siloxane copolymer has been used in this study. The molecular structure and phase transition temperatures of the PFLC are shown in Fig. 1. The detailed synthesis procedure and basic properties were previously reported. ${ }^{7}$ The mesogenic unit in the sidechain itself shows a large spontaneous polarization $P_{s}$ over $200 \mathrm{nC} / \mathrm{cm}^{2}$, so that the PFLC also shows a large $P_{s}$ of over $100 \mathrm{nC} / \mathrm{cm}^{2}$. As a guest compound,

${ }^{a)}$ Electronic mail: yoshino@ele.eng.osaka-u.ac.jp the dichroic dye NK-G241 (Nihon Kanko-Shikiso), with molecular structure given in Fig. 1, was used.

The PFLC and dichroic dye were dissolved in chloroform as a common solvent, and the solution was casted on the glass substrate with In-Sn oxide (ITO) electrodes by a spin-coating technique with rotation speed of $250 \mathrm{rpm}$ for 1 $\mathrm{s}$ and then $1500 \mathrm{rpm}$ for $15 \mathrm{~s}$. ITO electrodes were patterned with a gap of $1 \mathrm{~mm}$ on the surface of the substrate as shown in Fig. 2(a). The thickness of the electrodes was $200 \mathrm{~nm}$. Uniform homeotropically aligned film with smectic layers parallel to the substrate can be easily obtained by means of heating the spin-coated film to isotropic phase and cooling back to the chiral smectic $\mathrm{C}$ ( $\mathrm{SmC}^{*}$ ) phase. The good alignment was confirmed by polarizing microscopy. The thickness of the spin-coated film can be controlled by changing the concentration of solution and the rotation rate of spin coater. The typical thickness of the spin-coated PFLC layer used in this study is $2-3 \mu \mathrm{m}$.

For the optical absorption measurements, diode array multichannel spectrometer (IMUC-7000G, Otsuka electronics) with a polarizing microscope (Olympus) was used. As light source for the electrooptic measurements, a He-Ne laser $(632.8 \mathrm{~nm})$ was used. The transmitted light through the cell of the PFLC film was monitored by a photomultiplier.

A dichroic dye has an anisotropy in optical absorption, and its absorption depends on the light polarization direction

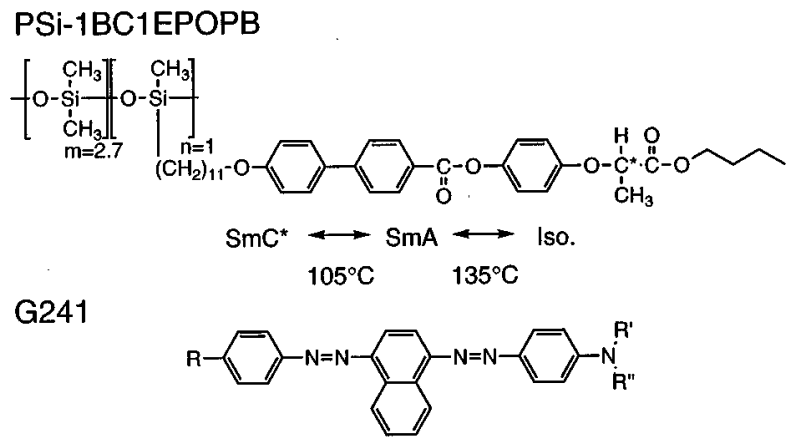

FIG. 1. Molecular structure and phase transition temperatures of the polymer ferroelectric liquid crystal and the dichroic dye used in this study. 


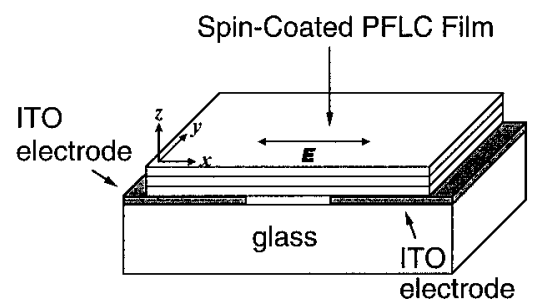

(a)
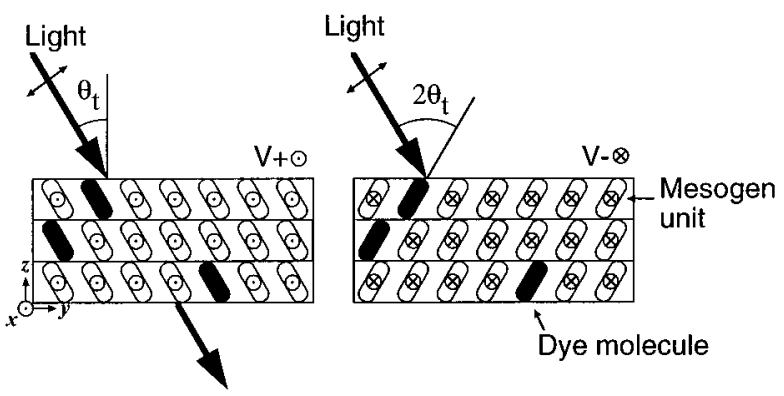

(b)

FIG. 2. (a) The cell configuration for the spin-coated device proposed in this study. (b) Schematic explanation of the guest-host electro-optic effect in a homeotropically aligned ferroelectric liquid crystal film.

relative to the molecular long axis. Using a liquid crystal doped with a dichroic dye, one can control the alignment of the dye molecules by the electric field in connection with the realignment of liquid crystal molecules. Therefore, in this system, a guest-host electro-optic effect can be realized, that is, the absorption of light can be controlled by the applied electric field.

In the cell configuration shown in Fig. 2(b), the electric field is applied parallel to the smectic layers along the $x$ axis and the director is tilted by a tilt angle $\theta_{t}$ with respect to the $z$ axis in the $y z$ plane perpendicular to the field. If the light propagates at the angle of $\theta_{t}$ to the layer normal ( $z$ axis), the propagation direction of the light is parallel to the molecular long axis of the liquid crystal in the film. When the polarity of the voltage is inverted, the tilt direction of the molecules reverses with respect to the $z$ axis and the light propagation direction makes an angle of $2 \theta_{t}$ to the molecular long axis. Assuming that the polarization direction of the light is in the $y z$ plane, under a positive voltage, $V^{+}$, the light propagates along the long molecular axis and the polarization of the light is perpendicular to the transition moment of the dye molecules. As a result, the light is not absorbed by the dyes and is transmitted through the film. On the other hand, under the application of $V^{-}$, the polarization direction of the light is almost parallel to the transition moment of the dye and the light is strongly absorbed.

The incident angle $\theta_{\text {in }}$ of the light is determined using the relation $\theta_{\text {in }}=\sin ^{-1}\left(n_{f} \sin \theta_{t}\right)$, where $n_{f}$ is the refractive index of the film. From Ref. 7, the tilt angle $\theta_{t}$ is $26^{\circ}$ and $23^{\circ}$ of $T=90^{\circ} \mathrm{C}$ and $100^{\circ} \mathrm{C}$, respectively.

Figure 3 shows absorption spectra of the spin-coated guest-host film upon the application of +100 and $-100 \mathrm{~V}$. When a positive field is applied, dye molecules align parallel to the propagating light, according to the host liquid crystal alignment, as shown in Fig. 2(b). As mentioned above, in

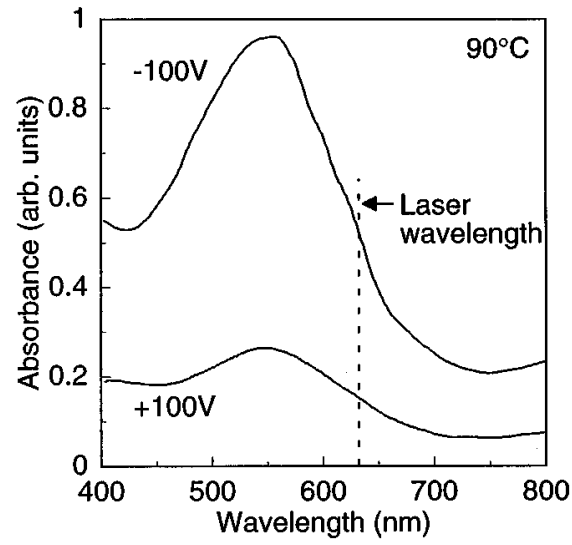

FIG. 3. Absorption spectra of the spin-coated guest-host PFLC film upon the application of +100 and $-100 \mathrm{~V}$.

this case, the polarization direction of light coincides with the direction of a short axis of dye molecules and is perpendicular to the transition moment. Therefore, the absorption in the dye molecules is small, resulting in a colorless state, as shown in Fig. 3. On the other hand, when an electric field of opposite polarity is applied, dye molecules make the angle $2 \theta_{t}$ with the direction of light, following the reorientation of liquid crystal molecules. In this state, the polarization of the light is nearly parallel to the transition moment of dye molecules. Consequently, the light is absorbed by the dye molecules and the transmitted light is colored, as shown in Fig. 3 . This shows that the guest-host electro-optic switching can be realized by means of the reorientation of dye molecules induced by guest-host interaction with the mesogenic units in the spin-coated polymer film.

A typical response of the transmitted light to the applied voltage is shown in Fig. 4. The change in transmission light intensity corresponds well to the polarity reversal of the field. The contrast ratio between on and off states estimated from Fig. 4 is about 3. In this study, a $\mathrm{He}-\mathrm{Ne}$ laser was used, with a wavelength $(632.8 \mathrm{~nm})$ not suitable to the dichroic dye, as is evident from Fig. 3. Higher contrast is expected by choosing a light source of wavelength at the absorption maximum of the dichroic dye. Indeed, a response with higher contrast was confirmed by the microscopic observation in which white light was used.

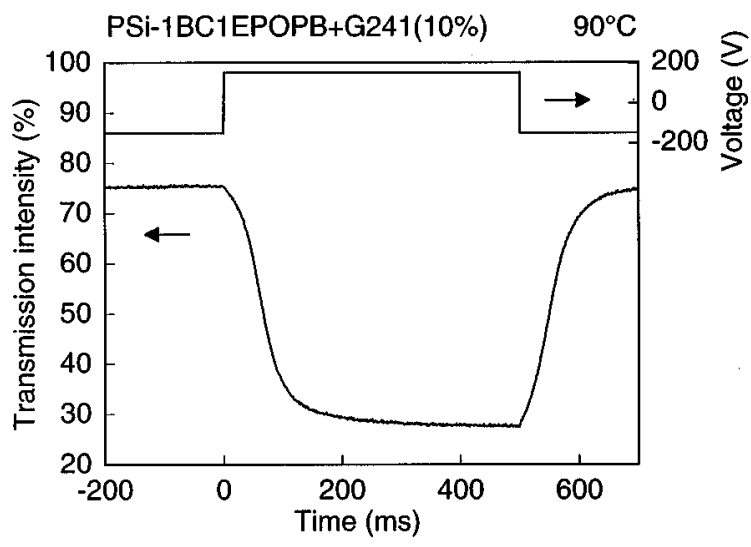

FIG. 4. Wave form of the optical response to a polarity reversal of the applied voltage in the spin-coated PFLC film. 


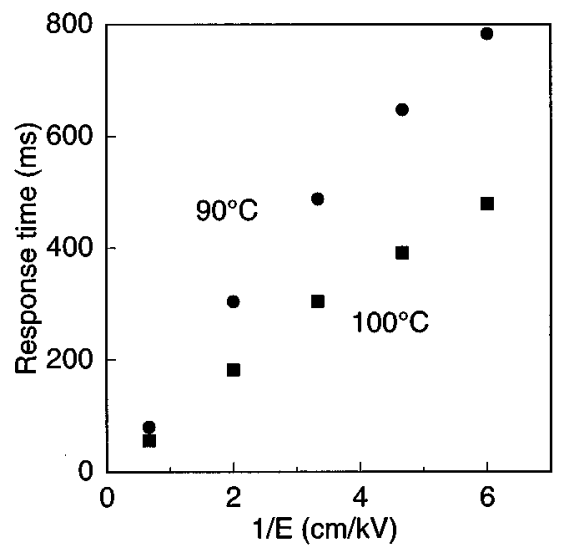

FIG. 5. Field dependence of the response time in the spin-coated PFLC film.

Figure 5 shows the voltage dependence of the response time in the spin-coated guest-host film. The response time is defined as the time required for the transmission intensity to change from $10 \%$ to $90 \%$ of the total transmission change. As is evident from this figure, the response time is proportional to the reciprocal of the applied voltage. In general, the driving force in FLC electro-optic devices with a macroscopic polarization originates from the interaction of $P_{s}$ and the electric field $E, P_{s} E$, and the response time. $\tau$ will be determined by the equation,

$$
\tau=\frac{\gamma}{P_{s} E},
$$

where $\gamma$ is the rotational viscosity. From this equation, $\tau$ should be inversely proportional to $P_{s}$ and $E$. The results in Fig. 5 suggest that, also in a spin-coated guest-host polymer film, $P_{s} E$ torque is the dominant driving force for the molecular reorientation in the electric field.
In the electrode configuration used in this study, the gap between electrodes is big and the effective electric field is not so high. Therefore, a much shorter response time can be realized by using a smaller electrode gap, such as an interdigitated electrode pattern. Estimating from the result in Fig. 5 , if the electrode gap is $5 \mu \mathrm{m}$, a response time around 2.3 ms can be obtained at the applied voltage of $20 \mathrm{~V}$.

We have proposed a new guest-host-type electro-optic effect in a free-surface thin film of polymer ferroelectric liquid crystals which is easily fabricated by a spin-coating technique. Combining this PFLC film with optical elements such as a prism and a waveguide, novel electro-optic devices can be designed. Since the spin-coated PFLC technique enables us to easily realize electro-optic thin polymer films, the application potential of this film technique must be very large.

This study was partly supported by a Grant-in-Aid for Science Research on Priority Areas from the Ministry of Education, Science, Sports and Culture and by the Swedish National Science Research Council.

${ }^{1}$ V. P. Shibaev, M. V. Kozlovsky, L. A. Beresnev, L. M. Blinov, and A. Plate, Polym. Bull. (Berlin) 12, 299 (1984).

${ }^{2}$ K. Yuasa, S. Uchida, T. Sekiya, K. Hashimoto, and K. Kawasaki, Ferroelectrics 122, 53 (1991)

${ }^{3}$ K. Skarp, K. Myojin, H. Moritake, M. Ozaki, K. Yoshino, and B. Helgee, J. Soc. Electr. Eng. 3, 69 (1994).

${ }^{4}$ K. Skarp, S. Uto, K. Myojin, H. Moritake, M. Ozaki, B. Helgee, and K. Yoshino, Jpn. J. Appl. Phys., Part 1 34, 5433 (1995).

${ }^{5}$ S. Uto, H. Moritake, M. Ozaki, K. Yoshino, K. Skarp, and B. Helgee, J. Appl. Phys. 79, 4444 (1996).

${ }^{6}$ S. Uto, H. Moritake, K. Myojin, M. Ozaki, K. Yoshino, K. Skarp, and B. Helgee, Ferroelectrics 181, 297 (1996).

${ }^{7}$ B. Helgee, T. Hjertberg, K. Skarp, G. Anderson, and F. Gouda, Liq. Cryst. 18, 871 (1995). 